Journal of Mathematics and Statistics 3(1): 28-35, 2007

ISSN 1549-3644

(C) 2007 Science Publications

\title{
Two Main Methods for Utility Sharing in Joint Business: A Discussion
}

\author{
Rafel Amer, Francesc Carreras and Antonio Magaña \\ Department of Applied Mathematics II, Industrial and Aeronautical Engineering School of Terrassa \\ Technical University of Catalonia, Spain
}

\begin{abstract}
One of the main purposes of any theory of joint business is to provide a convincing way for sharing the total utility available to the set of agents. The proportional rule is widely applied in practice but people using this method should be warned that it is not as innocent, effective, fair and consistent as it seems at first glance. Indeed, restricted domain, low sensibility derived from ignoring most marginal contributions, unjustified double discriminatory level and lack of additivity that derives in serious inconsistencies in costs/savings and added costs problems form part of the negative baggage of this rule, a fact that will probably surprise theorists and practitioners. Instead, nothing of this concerns the Shapley value whence the main conclusion of our analysis, which we hope the readers will find of interest, is that the Shapley value should replace the proportional rule in cooperative affairs and pure bargaining problems.
\end{abstract}

Key words: Cooperative game, proportional rule, Shapley value

\section{INTRODUCTION}

The proportional rule has a long tradition in collective problems where some kind of utility (costs, profits, savings...) is to be shared among the agents. However, while its (apparent) simplicity might seem a reason for applying it in pure bargaining affairs, where only the whole and the individual utilities matter, it is much more questionable in the case of general cooperative problems, where all marginal contributions should be taken into account. The axiomatic foundation proposed by Lloyd S. Shapley, when introducing the value notion for cooperative games, enables us to discuss positive and negative aspects of the proportional rule.

We will contrast the proportional rule with the Shapley value in this kind of problems and show, moreover, that pure bargaining affairs can be viewed as particular cases of cooperative affairs by introducing a suitable cooperative game closely attached to each one of them, so that the replacement of the proportional rule with the Shapley value would be convenient even in the pure bargaining case.

\section{COOPERATIVE AFFAIRS}

Let $N=\{1,2, \ldots, n\}$ be a set of agents (players). Each subset $S \subseteq N$ is a coalition. A cooperative game on $N$ (a game, for short, in the sequel) is a function $u$ that assigns to each coalition $S$ a real number $u(S)$, which is interpreted as the worth or utility that coalition $S$ is able to obtain independently of the behavior of the outside players (the members of $N \backslash S$ ). The only condition imposed to $u$ is that $u(\varnothing)=0$, where $\varnothing$ denotes the empty coalition. Nevertheless, one often deals with particular classes of games: a game $u$ is

* nonnegative if $u(S) \geq 0$ for every coalition $S$

* monotonic if $u(S) \leq u(T)$ whenever $S \subset T$

* super-additive if $u(S \cup T) \geq u(S)+u(T)$ whenever $S \cap T=\varnothing$

* additive if $u(S \cup T)=u(S)+u(T) \quad$ whenever $S \cap T=\varnothing$

* sub-additive if $u(S \cup T) \leq u(S)+u(T)$ whenever $S \cap T=\varnothing$

* symmetric if $u(S)$ depends only on the cardinality of $S$ for all $S \subseteq N$.

Usually, $u$ represents profits or savings arising from cooperation between the members of any coalition. In other cases, $u$ may well represent costs: we will then prefer to denote as $c$ this function. Rather than on the individual strategic possibilities of the agents, the cooperative theory is merely based on the amounts of utility that coalitions can get. The main question related to the problems represented by

Corresponding Author: $\quad$ Francesc Carreras, ETSEIAT, P.O. Box 577, E-08220 Terrassa, Spain 
cooperative games is the way to share among the players, in a rational way, the total utility of the grand coalition, that is, $u(N)$, in such a manner that all players agree and become (more or less) satisfied with the outcome. In this respect, an essential notion, of great relevance in economics, should be the following: the marginal contribution of a player $i \in N$ in a game $u$ to a coalition $S$ containing $i$ is $u(S)-u(S \backslash\{i\})$.

It will also be of interest to have in mind that $i \in N$ is a null player in a game $u$ on $N$ if all his marginal contributions vanish, i.e. if $u(S \cup\{i\})=u(S)$ for every $S \subseteq N \backslash\{i\}$, and $i, j \in N$ are equivalent players in a game $u$ on $N$ if their marginal contributions to each coalition coincide, i.e. if $u(S \cup\{i\})=u(S \cup\{j\})$ for every $S \subseteq N \backslash\{i, j\}$. The set $G_{N}$ of all cooperative games on a given set of players $N$ becomes a vector space of dimension $2^{n}-1$ endowed with the natural linear operations for realvalued functions defined by $(u+v)(S)=u(S)+v(S)$ and $(\lambda u)(S)=\lambda u(S)$ for all $S \subseteq N, u, v \in G_{N}$ and $\lambda \in \mathbb{R}$. We will pay special attention to the sum of games.

Example 2.1: Assume that three towns, A, B and C, wish to get some kind of supply (electricity, water, gas) from a supplier $\mathrm{S}$. The locations are $\mathrm{A}(2,2), \mathrm{B}(-2,2)$, $\mathrm{C}(-2,-2)$ and $\mathrm{S}(2,0)$, the distances being given in kilometres (Fig. 1). The connection cost amounts to 100 monetary units per $\mathrm{km}$.

For individual connections, the supplier offers lines SA, SB and SC. When A and B ask for the possibility of a joint connection, the supplier offers using SA and $\mathrm{AB}$, which represent a connection less expensive than $\mathrm{SA}$ and SB. Instead, for $\mathrm{A}$ and $\mathrm{C}$ the offer is just SA and SC. For B and $\mathrm{C}$, the supplier offers $\mathrm{SO}, \mathrm{OB}$ and $\mathrm{OC}$, where $\mathrm{O}$ denotes the origin. Finally, for $\mathrm{A}, \mathrm{B}$ and $\mathrm{C}$ together, the offer is using SA, SO, OB and OC. The problem will be how to share the connection costs.

Then we have a cost game $c$ on $N=\{1,2,3\}$, where 1 is $A, 2$ is $B$ and 3 is $C$, which states the costs derived from forming coalitions (including singletons) and signing contracts for establishing the necessary (either individual or joint) connections from the supplier to the towns and is given by $c(\varnothing)=0$ and

$$
\begin{aligned}
& c(\{1\})=200, \quad c(\{2\})=448, \quad c(\{3\})=448, \\
& c(\{1,2\})=600, \quad c(\{1,3\})=648, \quad c(\{2,3\})=766 \\
& \text { and } c(\{1,2,3\})=966 \text {. }
\end{aligned}
$$

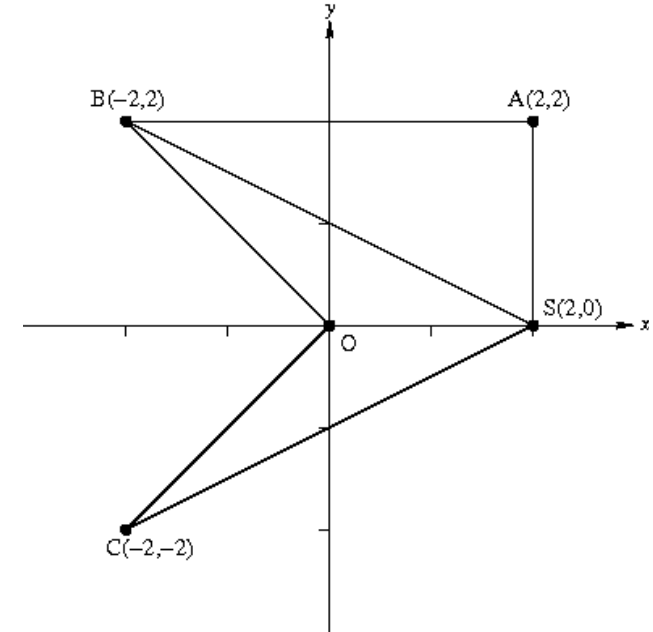

Fig. 1: Towns and supplier positions

Assume that the three towns sign a joint contract with the supplier. How should they share the total cost of 966? If, for example, only towns 1 and 2 sign a contract, how should they share their joint cost of 600 ?

Let us also consider an equivalent approach provided by considering the saving game $u$, which gives the savings derived from forming coalitions and is defined by

$u(S)=\sum_{i \in S} c(\{i\})-c(S)$ for each $S \subseteq N$.

In our example, $u(\varnothing)=0$ and

$$
\begin{array}{rlrl}
u(\{1\})=0, \quad u(\{2\})=0, & & u(\{3\})=0, \\
u(\{1,2\})=48, & u(\{1,3\})=0, & & u(\{2,3\})=130 \\
\text { and } u(\{1,2,3\})=130 . &
\end{array}
$$

Notice that both $c$ and $u$ are nonnegative and monotonic and $c$ is sub-additive whereas $u$ is superadditive. Now the questions are: how should the three towns share the net saving of 130 for a whole contract, or towns 1 and 2 share the net saving of 48 in the second case mentioned above? Of course, a coherent solution should exist for both cost and saving (related) problems.

\section{THE PROPORTIONAL RULE}

A well-known method to share $u(N)$ or $c(N)$ is given by the so-called proportional rule. The amount that this rule allocates to each agent $i \in N$ in, say, game $u$ is given by

$\pi_{i}[u]=\frac{u(\{i\})}{u(\{1\})+u(\{2\})+\ldots+u(\{n\})} u(N)$ 
Although an obvious "advantage" of the proportional rule is the easiness of its calculation in practice, a first problem arises as long as the rule can be applied only if

$u(\{1\})+u(\{2\})+\ldots+u(\{n\}) \neq 0$

so that its domain is limited to the class of cooperative games on $N$ that satisfy this condition. This is by no means a trivial restriction.

Example 3.1: In the case of the 3-member contract in Example 2.1, by applying formula (1) to game $c$ the proportional rule gives the sharing of the total cost of 966:

$\pi_{1}[c] \simeq 176.277, \pi_{2}[c] \simeq 394.861$ and $\pi_{3}[c] \simeq 394.861$.

Notice that: (a) towns 2 and 3 get the same cost allocation although they are not equivalent players in game $c$ because their individual costs coincide but 2 is an interesting partner for 1 whereas 3 is not and this reflects a lack of strategic sensibility of the proportional rule; (b) the proportional rule cannot be applied to game $u$ since all individual utilities vanish in this game and no proportion is therefore possible to be applied, thus illustrating the restricted domain problem of this sharing method.

\section{AN AXIOMATIC APPROACH TO A VALUE NOTION}

In a seminal work $^{[1,2]}$, Shapley addressed the sharing problem in completely general terms and introduced the axiomatic method in game theory. Indeed, he stated the problem of finding a value, that is, a map

$$
f: G_{N} \rightarrow \mathbb{R}^{N}
$$

which would assign a payoff vector $f[u]=\left(f_{1}[u], f_{2}[u], \ldots, f_{n}[u]\right)$ to every game $u$ on $N$. This payoff vector should be viewed as the solution to the problem represented by game $u$.

Of course, there are infinitely many ways to define such a map. Shapley stated four appealing conditions that a value $f$ should satisfy. They are the following:

* Efficiency. $\sum_{i \in N} f_{i}[u]=u(N)$ for every $u \in G_{N}$.

* Null player property. If $i \in N$ is a null player in a game $u \in G_{N}$ then $f_{i}[u]=0$.

* Symmetry. If $i, j \in N$ are equivalent players in a game $u \in G_{N}$ then $f_{i}[u]=f_{j}[u]$.

* Additivity. For all $u, v \in G_{N}, f[u+v]=f[u]+f[v]$.

These properties deserve to be called "axioms" because of their elegant simplicity. It is hard to claim that they are not compelling... Efficiency means that the players are going to share the total amount available to them. The null player property states that if a player does not contribute anything to all coalitions to which belongs then this player must get 0 . Symmetry establishes that two players that are equally interesting as coalition partners should receive the same payoff. Finally, additivity implies that the allocation in the sum game has to coincide with the sum of allocations in each game. Maybe, in spite of its simplicity and mathematical tradition, this latter property is, in principle, the least clear one: the reason is that one does not easily capture the meaning of the sum game in practice.

In his study, Shapley proved that there is one and only one function $f$ that satisfies these four axioms (which, moreover, form a logically independent system). He denoted it by $\phi$ and found that its expression is, for arbitrary $i \in N$ and $u \in G_{N}$,

$\phi_{i}[u]=\sum_{S \subseteq N: i \in S} \frac{(s-1) !(n-s) !}{n !}[u(S)-u(S \backslash\{i\})]$

where $s=|S|$ for each $S \subseteq N$.

Of course, the subsequent literature has been referring to $\phi$ as the Shapley value. Other not essential but interesting properties follow from the axioms. For example, positivity: if a game $u$ is monotonic, then $\phi_{i}[u] \geq 0$ for each $i \in N$. Or: if $u$ is super-additive, then the Shapley value is an imputation in the sense that, jointly with efficiency, we have $\phi_{i}[u] \geq u(\{i\})$ for all $i \in N$. Notice moreover that, in fact, the Shapley value becomes a linear map from $G_{N}$ to $\mathbb{R}^{N}$ since $\phi[\lambda u]=\lambda \phi[u]$ for all $u \in G_{N}$ and all $\lambda \in \mathbb{R}$.

Also notice that, on one hand, the calculus for the Shapley value is more laborious than for the proportional rule. Nevertheless, computer programs exist that provide the Shapley value of a game and the multi-linear extension of a game $^{[3]}$ becomes an interesting tool for using, if necessary, approximation methods. On the other hand, all marginal contributions of a player matter when computing the Shapley value for this player. This implies a great deal of strategic sensibility on the side of the Shapley value that should be highly appreciated by practitioners and will be illustrated by all our following examples.

Example 4.1: In the case of the 3-member contract in Example 2.1, the Shapley value gives, using formula (3), 


$$
\begin{aligned}
& \phi_{1}[c]=\frac{200}{3}+\frac{152}{6}+\frac{200}{6}+\frac{200}{3}=\frac{1152}{6}=192, \\
& \phi_{2}[c]=\frac{448}{3}+\frac{400}{6}+\frac{318}{6}+\frac{318}{3}=\frac{2250}{6}=375 \\
& \phi_{3}[c]=\frac{448}{3}+\frac{448}{6}+\frac{318}{6}+\frac{366}{3}=\frac{2394}{6}=399
\end{aligned}
$$

so that: (a) as should be expected, the Shapley value discriminates between 2 and 3 by taking into account all marginal contributions of each town; (b) analogously, it perfectly applies to the saving game $u$ and yields

$\phi_{1}[u]=8, \phi_{2}[u]=73$, and $\phi_{3}[u]=49$;

and (c) it is coherent in the sense that, for every town $i$, $\phi_{i}[c]+\phi_{i}[u]=c(\{i\})$

(that's why town 2 gets more profit than town 3 in game $u$ ). The conclusion is that, using the Shapley value, all towns are indifferent between sharing costs and sharing savings, as it should be.

Example 4.2: Let $u$ be the nonnegative and superadditive (and hence monotonic) game defined by $u(\varnothing)=0$ and $u(\{1\})=1, u(\{2\})=2, u(\{3\})=0$,

$u(\{1,2\})=6, u(\{1,3\})=6, u(\{2,3\})=5$ and

$u(\{1,2,3\})=11$.

Notice that player 3 is not null and players 1 and 2 are not equivalent in this game. The proportional rule gives $\pi_{1}[u] \simeq 3.67, \pi_{2}[u] \simeq 7.33$ and $\pi_{3}[u]=0.00$

whereas the Shapley value yields

$\phi_{1}[u]=4, \phi_{2}[u]=4$ and $\phi_{3}[u]=3$.

Although in this instance both allocation rules are imputations, the striking differences arise from the fact that the proportional rule completely ignore (the marginal contributions of the players to) the coalitions of size 2 .

\section{CHECKING THE PROPORTIONAL RULE}

The framework established by Shapley allows us to evaluate any allocation method and, in particular, to compare the proportional rule and the Shapley value. The relevant points are the following:

I. As was pointed out before, a first essential failure of the proportional rule is its restricted domain, defined by condition (2). Instead, the Shapley value applies without any restriction to all cooperative games.

II. A second important problem is that the proportional rule does not take into account most of the marginal contributions (see Examples 3.1 and 4.2); this becomes more and more critical as the number of players increases and results in the very low sensibility already mentioned. On the contrary, the Shapley value is always concerned with all marginal contributions without exception and enjoys therefore a nice sensibility with regard to the problem data.

III. It is instructive to put together formulas (1) and (3) for two-player games. If $i$ and $j$ are these players (so that $\{i, j\}=\{1,2\}$ ) then we have

$$
\pi_{i}[u]=u(\{i\})+\frac{u(\{i\})}{u(\{i\})+u(\{j\})}[u(\{i, j\})-u(\{i\})-u(\{j\})]
$$

and

$$
\phi_{i}[u]=u(\{i\})+\frac{1}{2}[u(\{i, j\})-u(\{i\})-u(\{j\})]
$$

The procedures look partially similar: first, each player is allocated his claim (say, $u(\{i\})$ in case of player $i$ ); then, the remaining worth is shared among both. However, it is worthy of mention that the Shapley value simply shares the residual utility equitably, whereas the proportional rule shares it proportionally to the individual utilities. This means that, conceptually, the proportional rule is more complicated than the Shapley value and includes a double discriminatory level that rewards twice the player that individually can get the highest utility by his own. We fail to find a reasonable justification for this. The use of formulas (4) and (5) is illustrated in Example 5.1 and an interesting generalization of the phenomenon is presented in Example 5.2.

IV. In which cases do both allocation rules coincide? It is not difficult to see that, in the two-player case, the Shapley value and the proportional rule coincide on a game $u$ (such that $u(\{1\})+u(\{2\}) \neq 0$, of course) if and only if this game satisfies some of the following conditions:

(i) $u(\{1\})=u(\{2\})$ (symmetric game).

(ii) $u(\{1,2\})=u(\{1\})+u(\{2\})$ (additive game).

In general (arbitrary $n$ ), for any additive game $u$ satisfying (2) we have $\phi[u]=\pi[u]$ (in fact, the $i$-component of both values coincides with $u(\{i\})$ for each $i \in N)$. Also, for any symmetric game $u$ satisfying (2) we get $\phi_{i}[u]=\frac{u(N)}{n}=\pi_{i}[u]$ for all $i \in N$.

V. As to Shapley's axioms, it is easy to check that, in its restricted domain defined by inequality (2), the 
proportional rule satisfies the axioms of efficiency, null player and symmetry. Even positivity is satisfied and the proportional rule gives imputations for super-additive games.

VI. This leaves us with the lack of additivity for this rule (otherwise, it would coincide with the Shapley value by the uniqueness of the value). Then, although the proportional rule satisfies $\pi[\lambda u]=\lambda \pi[u]$ for all game $u$ in its domain and all $\lambda \in \mathbb{R}$, the proportional rule is not linear. Let us raise the following question: is this failure important or, on the contrary, additivity is simply a standard mathematical property, just of a technical nature, without special relevance for practitioners? The answer is quite surprising. From the lack of additivity, serious inconsistencies of the proportional rule derive when applying it to certain problems. Examples 5.3 and 5.4 will illustrate this.

Example 5.1: Going back to Example 2.1, let us consider the case where only towns 1 and 2 sign a joint contract with the supplier. Then only coalitions excluding town 3 have to be taken into account and this leads us to the restricted games $c^{\prime}$ and $u^{\prime}$ defined on $N^{\prime}=\{1,2\}$ as follows:

$c^{\prime}(\{1\})=200, \quad c^{\prime}(\{2\})=448, \quad c^{\prime}(\{1,2\})=600$, $u^{\prime}(\{1\})=0, \quad u^{\prime}(\{2\})=0, \quad u^{\prime}(\{1,2\})=48$,

and, of course, $c^{\prime}(\varnothing)=0$ and $u^{\prime}(\varnothing)=0$. By applying e.g. formulas (4) and (5), we get $\pi_{1}\left[c^{\prime}\right] \simeq 181.185$ and $\pi_{2}\left[c^{\prime}\right] \simeq 414.815$ but no possibility to apply the proportional rule to $u^{\prime}$; instead,

$\phi_{1}\left[c^{\prime}\right]=176, \phi_{2}\left[c^{\prime}\right]=424, \phi_{1}\left[u^{\prime}\right]=24$ and $\phi_{2}\left[u^{\prime}\right]=24$, so that $\phi$ is, again, coherent: $\phi_{i}\left[c^{\prime}\right]+\phi_{i}\left[u^{\prime}\right]=c^{\prime}(\{i\})$ for $i=1,2$.

Example 5.2: A pure bargaining problem for a set of agents $N$ can be described by a set of utilities $u_{1}, u_{2}, \ldots, u_{n}$ available to the agents individually and a total utility $u_{N}$ they can jointly get by cooperating. As we will see, not only the proportional rule but also the Shapley value can be applied to share $u_{N}$. To this end, we need to introduce a cooperative game.

Since nothing is said about the utility available to each intermediate coalition, we can reasonably assume that such a coalition can get the sum of individual utilities of its members. Then we define the quasiadditive closure of the pure bargaining problem as the cooperative game $\bar{u}$ on $N$ given by
$\bar{u}(N)=u_{N}$ and $\bar{u}(S)=\sum_{i \in S} u_{i}$ for $S \subset N$.

Notice that $\bar{u}(\{i\})=u_{i}$ for all $i \in N$. In general, game $\bar{u}$ is not additive because $u_{N}$ needs not coincide with $u_{1}+u_{2}+\ldots+u_{n}$. An elementary calculus shows that the generalizations of (4) and (5) hold, i.e.

$\pi_{i}[\bar{u}]=u_{i}+\frac{u_{i}}{\sum_{j \in N} u_{j}}\left[u_{N}-\sum_{j \in N} u_{j}\right]$

and

$\phi_{i}[\bar{u}]=u_{i}+\frac{1}{n}\left[u_{N}-\sum_{j \in N} u_{j}\right]$,

using, in the second case, the well known fact that

$\sum_{S \subseteq N: i \in S} \frac{(s-1) !(n-s) !}{n !}=1$ for each $i \in N$.

Therefore we find again an unjustified double discriminatory behavior of the proportional rule that contrasts with the simplicity of the Shapley value. In addition, it is shown that the Shapley value (applied to the quasi-additive closure) is also a better solution even for pure bargaining problems.

Example 5.3: Let $N=\{1,2,3\}$ be a purchasing pool of three firms and assume that, periodically, its members make, to a common supplier, orders of 1000, 1500 and 2000 units, respectively, of a product whose unitary cost is 1 . The supplier offers the following abseiling:

nothing from 1 to 999 units

$10 \%$ off from 1000 to 1999 units

$15 \%$ off from 2000 to 2999 units

$20 \%$ off from 3000 upwards.

Table 1 provides the full data for this purchasing pool. The members of the pool do not form a joint venture. They join just to get discounts for accumulated orders. Two alternatives are offered: (a) sharing the actual joint cost of 3600; (b) sharing the joint saving of 900 after assuming that, previously, all members have individually deposited in a joint bank account the cost of their respective orders without discounts. Table 2 provides the result of applying the proportional rule and the Shapley value to each alternative.

Notice that we have three games: an additive game $c$ of costs without discount, game $c^{d}$ of costs with discount and game $u$ of savings. They are obviously related by $c=c^{d}+u$. While the Shapley value is consistent in the sense that $\phi[c]=\phi\left[c^{d}\right]+\phi[u]$, which follows by additivity from $c=c^{d}+u$, this is not the 
Table 1: Purchasing pool data

\begin{tabular}{lllll}
\hline group & $\begin{array}{l}\text { order } \\
c\end{array}$ & discount & $\begin{array}{l}\text { real cost } \\
c^{d}\end{array}$ & $\begin{array}{l}\text { saving } \\
u\end{array}$ \\
\hline$\{1\}$ & 1000 & $10 \%$ & 900 & 100 \\
$\{2\}$ & 1500 & $10 \%$ & 1350 & 150 \\
$\{3\}$ & 2000 & $15 \%$ & 1700 & 300 \\
$\{1,2\}$ & 2500 & $15 \%$ & 2125 & 375 \\
$\{1,3\}$ & 3000 & $20 \%$ & 2400 & 600 \\
$\{2,3\}$ & 3500 & $20 \%$ & 2800 & 700 \\
$\{1,2,3\}$ & 4500 & $20 \%$ & 3600 & 900 \\
\hline
\end{tabular}

Table 2: Purchasing pool allocations

\begin{tabular}{lcccccc}
\hline$i$ & $\pi_{i}[c]$ & $\pi_{i}\left[c^{d}\right]$ & $\pi_{i}[u]$ & $\phi_{i}[c]$ & $\phi_{i}\left[c^{d}\right]$ & $\phi_{i}[u]$ \\
\hline 1 & 1000 & 820.25 & 163.64 & 1000 & 812.50 & 187.50 \\
2 & 1500 & 1230.38 & 245.45 & 1500 & 1237.50 & 262.50 \\
3 & 2000 & 1549.37 & 490.91 & 2000 & 1550.00 & 450.00 \\
sums & 4500 & 3600.00 & 900.00 & 4500 & 3600.00 & 900.00 \\
\hline
\end{tabular}

case for the proportional rule, which does not satisfy this property as can be checked in Table 2. Notice, moreover, that $\pi[c]=\phi[c]$ because $c$ is an additive game.

Therefore, when using the Shapley value all members of the pool are indifferent between sharing costs with discount and sharing savings. Instead, this is not the case if the proportional rule is applied: firms 1 and 2 prefer sharing costs whereas firm 3 prefers sharing savings: the inconsistency (or lack of fairness) of the procedure is obvious.

Example 5.4: Let us assume now that Example 2.1 was referring to e.g. gas supply and the towns are also interested in an additional water supply (carried out by the same supplier), the cost of which is given in (the second column of) Table 3.

Again, we consider three games: the additive and nonnegative (and hence monotonic) game $c_{w}$, where 1 is a null player and 2 and 3 are equivalent players, describes the water costs; game $c_{g}$ (equivalent to game $c$ in Example 2.1) gives the gas costs; and the sum game $c_{w}+c_{g}$ yields the added costs. Table 4 provides the result of applying the proportional rule and the Shapley value to each one of these games. Notice that the proportional rule and the Shapley value coincide on $c_{w}$ since this is an additive game. While the Shapley value is consistent in the sense that $\phi\left[c_{w}+c_{g}\right]=\phi\left[c_{w}\right]+\phi\left[c_{g}\right]$ and this follows from additivity, this is not the case for the proportional rule, which does not satisfy this property and fails therefore to be consistent in added costs problems.

In this case, if the proportional rule is applied, town 1 prefers to share the payment of separate bills whereas towns 2 and 3 prefer to share the payment of a water + gas joint bill. Instead, by using the Shapley value, all

\begin{tabular}{lcll} 
Table 3: & Water and gas supply & & \\
\hline group & water & gas & water + gas \\
& costs & costs & costs \\
& $c_{\mathrm{w}}$ & $\mathrm{c}_{\mathrm{g}}$ & $\mathrm{c}_{\mathrm{w}}+\mathrm{c}_{\mathrm{g}}$ \\
\hline$\{1\}$ & 0 & 200 & 200 \\
$\{2\}$ & 50 & 448 & 498 \\
$\{3\}$ & 50 & 448 & 498 \\
$\{1,2\}$ & 50 & 600 & 650 \\
$\{1,3\}$ & 50 & 648 & 698 \\
$\{2,3\}$ & 100 & 766 & 866 \\
$\{1,2,3\}$ & 100 & 966 & 1066 \\
$\{1\}+\{2\}+\{3\}$ & 100 & 1096 & 1196 \\
\hline
\end{tabular}

\begin{tabular}{lllllll}
\multicolumn{6}{l}{ Table 4: Water and gas allocations } \\
\hline$i$ & $\pi_{i}\left[c_{w}\right]$ & $\pi_{i}\left[c_{g}\right]$ & $\pi_{i}\left[c_{w}+c_{g}\right]$ & $\phi_{i}\left[c_{w}\right]$ & $\phi_{i}\left[c_{g}\right]$ & $\phi_{i}\left[c_{w}+c_{g}\right]$ \\
\hline 1 & 0 & 176.277 & 178.261 & 0 & 192 & 192 \\
2 & 50 & 394.861 & 443.870 & 50 & 375 & 425 \\
3 & 50 & 394.861 & 443.870 & 50 & 399 & 449 \\
& & & & & & \\
sums & 100 & 965.999 & 1066.001 & 100 & 966 & 1066 \\
\hline
\end{tabular}

towns are indifferent between sharing separate bills and sharing a joint bill.

\section{USE AS POWER INDICES}

An important class of cooperative games is formed by the so-called simple games. A game $u$ is simple if it is monotonic, $u(S)=0$ or 1 for every coalition $S$ and $u(N)=1$. Such a game is completely defined by the family of winning coalitions $W=W(u)=\{S \subseteq N: u(S)=1\}$.

Even more, the subfamily of minimal (in the inclusion sense) winning coalitions $W^{m}$ also determines the game. Simple games are frequently used to represent voting systems at all levels, such as shareholder corporations, participated firms, town councils, parliamentary bodies and supranational organizations and the notion of power has therefore been closely attached to them. Let $S G_{N}$ denote the set of all simple games on a given player set $N$. This set is not a linear subspace of $G_{N}$ but a sub-lattice under the standard composition laws $\vee$ and $\wedge$, which are given by $(u \vee v)(S)=\max \{u(S), v(S)\}$ and $(u \wedge v)(S)=\min \{u(S), v(S)\}$ for all $u, v \in S G_{N}$ and every $S \subseteq N$.

Although there is no general agreement on a single power index, among either game theorists or political scientists, there is an almost common view of power as a "cake" to be shared. Then, at least two properties are desirable for a map $g: S G_{N} \rightarrow \mathbb{R}^{N}$ to be considered as a suitable power index: 
$\sum_{i \in N} g_{i}[u]=1$ for all $u \in S G_{N}$

$g_{i}[u] \geq 0$ for all $i \in N$ and all $u \in S G_{N}$.

And indeed, the restriction of the Shapley value $\phi$ to $S G_{N}$ satisfies these properties: property 1 is nothing but efficiency, while property 2 derives from the positivity property of the Shapley value and the fact that any simple game is monotonic. Moreover, the null player property and symmetry have also a nice interpretation for simple games. In effect, it is easy to check that a player is null in a simple game if and only if this player does not belong to any minimal winning coalition. Then, it is only natural that such a player gets a power of 0 . Quite similarly, one can check that two players are equivalent in a simple game if and only if they appear symmetrically in the list of minimal winning coalitions. It is also completely reasonable that two such players have the same power. Thus, as was first pointed out by Shapley and Shubik ${ }^{[2,4]}$, the restriction of the Shapley value to the lattice of simple games behaves perfectly well as a power index. Since then, it is usually called the Shapley-Shubik index of power.

As to additivity, it makes no sense in simple games. However, Dubey ${ }^{[5]}$ discovered that an axiomatic characterization of the Shapley-Shubik power index, as, say, an allocation rule or "value" on the class of simple games, can be achieved by replacing that useless property with the so-called transfer property, which characterizes valuations in a lattice:

$g[u \vee v]=g[u]+g[v]-g[u \wedge v]$ for all $u, v \in S G_{N}$.

Although the proportional rule satisfies properties 1 and 2 above in its restricted domain, it is clearly unable as a power index since almost all simple games fail to satisfy condition (2) because, in such games, $u(\{i\})=0$ for all $i \in N$ unless $i$ is a dictator or a winner (very exceptional cases).

Instead, the Shapley-Shubik power index not only applies to all simple games but also shows an extreme sensibility to data changes, as is illustrated by the following example. Let us point out that, in view of conditions 1 and 2, the allocations given by the Shapley-Shubik power index can be expressed as percentages.

Example 6.1: Let us consider a participated firm where the share among four stockholders, denoted as 1, 2, 3 and 4 , is $36.25,34.50,15.50$ and $13.75 \%$, respectively. Assuming decisions are taken under the absolute majority rule, the game among the shareholders is the weighted majority game $u$ denoted by
Table 5: The Shapley-Shubik index of power in the participated firm

\begin{tabular}{lllrrrr}
\hline case & quota & stockholder & \multicolumn{1}{c}{1} & \multicolumn{1}{c}{2} & \multicolumn{1}{c}{3} & \multicolumn{1}{c}{4} \\
\hline$(0)$ & 50.01 & weight & 36.25 & 34.50 & 15.50 & 13.75 \\
& $(1 / 2)$ & power & 41.67 & 25.00 & 25.00 & 8.33 \\
$(1)$ & 50.01 & weight & 51.25 & 29.50 & 10.50 & 8.75 \\
& $(1 / 2)$ & power & 100.00 & 0.00 & 0.00 & 0.00 \\
$(2)$ & 50.01 & weight & 41.25 & 34.50 & 15.50 & 8.75 \\
& $(1 / 2)$ & power & 41.67 & 25.00 & 25.00 & 8.33 \\
$(3)$ & 50.01 & weight & 36.25 & 39.50 & 15.50 & 8.75 \\
& $(1 / 2)$ & power & 33.33 & 33.33 & 33.33 & 0.00 \\
$(4)$ & 60.00 & weight & 36.25 & 34.50 & 15.50 & 13.75 \\
& $(3 / 5)$ & power & 33.33 & 33.33 & 16.67 & 16.67 \\
$(5)$ & 66.67 & weight & 36.25 & 34.50 & 15.50 & 13.75 \\
& $(2 / 3)$ & power & 50.00 & 50.00 & 0.00 & 0.00 \\
\hline
\end{tabular}

$$
u \equiv[50.01 ; 36.25,34.50,15.50,13.75]
$$

This means that $u$ is a simple game where each agent $i$ has been allocated a weight of $w_{i} \geq 0$ (percentage of share in our case), there is a quota $q$ (50.01 in our case) and the winning coalitions are defined by

$S \in W=W(u)$ if and only if $\sum_{i \in S} w_{i} \geq q$.

Thus, in this example we have

$W^{m}=\{\{1,2\},\{1,3\},\{2,3,4\}\}$.

Game $u$ is super-additive. Starting at this initial situation, that will be denoted by (0) in Table 5, let us consider several variations, all of them defined by super-additive simple games:

(1) player 1 buys $5 \%$ of the share to each one of the remaining players

(2) only player 4 sells $5 \%$ of the share to player 1

(3) player 4 sells $5 \%$ of the share to player 2

(4) without purchase of stock, the majority is raised to $3 / 5$

(5) without purchase of stock, the majority is raised to $2 / 3$

Table 5 provides the Shapley-Shubik index of power for the original game and each one of the games arising from the above changes.

\section{CONCLUSION}

So far we have analyzed the proportional rule, from the axiomatic viewpoint established by Shapley when defining the value notion for cooperative games but also from a practical viewpoint. Several properties and failures of the proportional rule have been remarked (items I-VI and a statement previous to Example 6.1) and especially, practical implications of the nonadditivity of this rule have been evidenced that result in a serious inconsistency when dealing with costs/savings related problems and added costs problems. 
Summing up, it might be said that, in spite of its greater difficulty of calculus (easily solved by using computer programs or approximation methods for a high number of players), the Shapley value should replace in practice the proportional rule in cooperative affairs, where coalitions of intermediate size $(1<|S|<n)$ matter, but also in pure bargaining problems, as has been shown in Example 5.2.

We would like to end the study by mentioning several references. First, the material included here might be completed with references ${ }^{[6-8]}$, where additional information is provided. Applications of cooperative games to economic problems may be found $\mathrm{in}^{[9]}$ and $^{[10]}$ and even in (the chapters on cooperative games of $^{[11]}$, a great classical book on game theory. For an attempt to give to the proportional rule a greater relevance in cooperative games ${ }^{[12]}$.

\section{ACKNOWLEDGEMENTS}

Research partially supported by Grant MTM 200606064 of the Spanish Ministry of Education and Science and the European Regional Development Fund and Grant SGR 2005-00651 of the Catalonia Government (Generalitat de Catalunya).

\section{REFERENCES}

1. Shapley, L.S., 1953. A value for n-person games. Ann. Math. Studies, 28: 307-317.
2. Roth, A.E. (Ed.), 1988. The Shapley Value: Essays in Honour of Lloyd S. Shapley. Cambridge University Press.

3. Owen, G., 1972. Multi-linear extension of games. Management Sci., 18: 64-79.

4. Shapley, L.S. and M. Shubik, 1954. A method for evaluating the distribution of power in a committee system. Am. Polit. Sci. Rev., 48: 787-792.

5. Dubey, P., 1975. On the uniqueness of the Shapley value. Intl. J. Game Theory, 4: 131-139.

6. Amer, R., F. Carreras and A. Magaña, 2001. Game Theory (in Spanish). Edicions UPC.

7. Amer, R., F. Carreras and A. Magaña, 2003. Simple games and Shapley-Shubik power index (in Spanish). Revista de Estudios Políticos, 121: 107-136.

8. Amer, R., F. Carreras and A. Magaña, 2006. Cooperative games and Shapley value. (in Spanish). Forthcoming in Alta Dirección.

9. Bilbao, J.M. and F.R. Fernández (Eds.), 1999. Advances in game theory with economic and social applications (in Spanish). Publication Secretariat of the Sevilla University.

10. Rafels, C. (Coord.), 1999. Cooperative games and economic applications (in Catalan). University of Barcelona Editions.

11. Owen, G., 1995. Game Theory. Academic Press $\left(3^{\text {rd }}\right.$. edition).

12. Ortmann, K.M., 2000. The proportional value for positive cooperative games. Mathematical Methods of Operations Research, 51: 235-248. 